Erratum

\title{
Erratum: Crews, T.E.; Rumsey, B.E. What Agriculture Can Learn from Native Ecosystems in Building Soil Organic Matter: A Review. Sustainability 2017, 9, 578
}

Timothy E. Crews ${ }^{1, *}$ and Brian E. Rumsey ${ }^{2}$

1 The Land Institute, 2440 E. Water Well Rd., Salina, KS 67401, USA

2 Environmental History, University of Kansas, 3650 Wescoe Hall, Lawrence, KS 66045, USA; brian.rumsey@gmail.com

* Correspondence: crews@landinstitute.org; Tel.: +1-785-823-5376

Received: 19 March 2018; Accepted: 20 March 2018; Published: 22 March 2018

The authors would like to correct the reference numbers listed in Table 2 of their paper [1]. The reference numbers which need to be corrected are underlined in the original version of Table 2 . The original Table 2 reads as follows:

Table 2. Summary of field-based estimations of soil carbon accumulation rates in the conversion of annual agriculture to perennial grassland or perennial bioenergy crops.

\begin{tabular}{|c|c|c|c|c|c|}
\hline Study Type & Geographic Areas & $\begin{array}{c}\text { Mean C } \\
\text { Accumulation } \\
t_{\text {ha }} \mathbf{a}^{-1} \text { year }^{-1}\end{array}$ & $\begin{array}{c}\text { Depths }^{1} \\
\text { Sampled (cm) }\end{array}$ & $\begin{array}{l}\text { No. Studies or } \\
\text { Sites Included }\end{array}$ & Reference \\
\hline \multicolumn{6}{|c|}{ Annual crops to perennial pasture or restored native grassland } \\
\hline Meta-analysis & $\begin{array}{l}\text { Central Europe, } \\
\text { N. America, Russia }\end{array}$ & 0.72 & $0-30$ & 273 & $\underline{[93]}$ \\
\hline Meta-analysis & Russia & 0.96 & 20 & 45 & [95] \\
\hline Meta-analysis & Tropical to temperate & 0.33 & $5-300$ & 39 & [96] \\
\hline Meta-analysis & Americas, U.K., Australia & 1.01 & $\mathrm{NR}^{2}$ & 23 & [97] \\
\hline Review & N. Midwest USA & $0.44-0.5$ & 25 & 39 & {$[98]$} \\
\hline Review & W. Canada & 0.59 & NR & 17 & {$[99]$} \\
\hline Chronosequences & Illinois, USA & 0.43 & 100 & 16 & [100] \\
\hline Review & France & 0.50 & NR & - & {$[101]$} \\
\hline Review & NR & $0.3-1.0$ & NR & - & [102] \\
\hline \multicolumn{6}{|c|}{ Annual crops to perennial bioenergy crops } \\
\hline Meta-analysis & NR & $1.14-1.88$ & $0-150$ & 23 & [103] \\
\hline Meta-analysis & N. \& S. America, Europe & & & & \\
\hline Miscanthus & S. Africa, Asia & 1.09 & 100 & 13 & [89] \\
\hline Switchgrass & & 1.28 & 100 & 40 & [89] \\
\hline
\end{tabular}

\footnotetext{
${ }^{1}$ When a range is reported, it indicates that multiple soil depths falling within the range were included in the study;
}

${ }^{2} \mathrm{NR}=$ not reported. 
The corrected Table 2 reads as follows:

Table 2. Summary of field-based estimations of soil carbon accumulation rates in the conversion of annual agriculture to perennial grassland or perennial bioenergy crops.

\begin{tabular}{|c|c|c|c|c|c|}
\hline Study Type & Geographic Areas & $\begin{array}{c}\text { Mean C } \\
\text { Accumulation } \\
\mathrm{t} \mathrm{ha}^{-1} \text { year }^{-1}\end{array}$ & $\begin{array}{c}\text { Depths }^{1} \\
\text { Sampled (cm) }\end{array}$ & $\begin{array}{l}\text { No. Studies or } \\
\text { Sites Included }\end{array}$ & Reference \\
\hline \multicolumn{6}{|c|}{ Annual crops to perennial pasture or restored native grassland } \\
\hline Meta-analysis & $\begin{array}{c}\text { Central Europe, } \\
\text { N. America, Russia }\end{array}$ & 0.72 & $0-30$ & 273 & [89] \\
\hline Meta-analysis & Russia & 0.96 & 20 & 45 & [90] \\
\hline Meta-analysis & Tropical to temperate & 0.33 & $5-300$ & 39 & [91] \\
\hline Meta-analysis & Americas, U.K., Australia & 1.01 & $\mathrm{NR}^{2}$ & 23 & [92] \\
\hline Review & N. Midwest USA & $0.44-0.5$ & 25 & 39 & [93] \\
\hline Review & W. Canada & 0.59 & NR & 17 & [94] \\
\hline Chronosequences & Illinois, USA & 0.43 & 100 & 16 & [95] \\
\hline Review & France & 0.50 & NR & - & [96] \\
\hline Review & NR & $0.3-1.0$ & NR & - & [97] \\
\hline \multicolumn{6}{|c|}{ Annual crops to perennial bioenergy crops } \\
\hline Meta-analysis & NR & $1.14-1.88$ & $0-150$ & 23 & [98] \\
\hline Meta-analysis & N. \& S. America, Europe & & & & \\
\hline Miscanthus & S. Africa, Asia & 1.09 & 100 & 13 & [99] \\
\hline Switchgrass & & 1.28 & 100 & 40 & [99] \\
\hline
\end{tabular}

\section{Reference}

1. Crews, T.E.; Rumsey, B.E. What Agriculture Can Learn from Native Ecosystems in Building Soil Organic Matter: A Review. Sustainability 2017, 9, 578. [CrossRef]

(C) 2018 by the authors. Licensee MDPI, Basel, Switzerland. This article is an open access article distributed under the terms and conditions of the Creative Commons Attribution (CC BY) license (http:/ / creativecommons.org/licenses/by/4.0/). 